\title{
SEXISMO E DOCÊNCIA DO ENSINO SUPERIOR: ANÁLISE DA REPRESENTATIVIDADE FEMININA NA DOCÊNCIA DOS CURSOS DE EXATAS
}

Samara Peireira de Sousa, UFCG, samarapss@ gmail.com

\begin{abstract}
RESUMO
Este trabalho tem por objetivo analisar a representatividade feminina do corpo docente dos cursos de exatas, refletindo acerca das relações de gênero constituídas na sociedade que contribuem para uma desigualdade entre homens e mulheres. Para empreender tal discussão, utilizou-se como aporte teórico Barreto (2014), Butler (2012), Pinto e Cruz (2012), Lopes (2016), entre outros. Optou-se por uma pesquisa qualitativa de cunho bibliográfica interpretativa na busca de significações para as concepções teóricas expostas na literatura existente. As análises apontaram que apesar da inserção ao longo das últimas décadas de diversas políticas públicas direcionadas ao acesso do ensino superior, em especial aos cursos de exatas, a participação do público feminino em cursos dessa área ainda é bastante reduzida, mas que há uma crescente considerável e, por isso, pesquisas nesse sentido se tornam importantes no âmbito acadêmico para promover o debate e a reflexão acerca da desigualdade de gêneros existente em nosso meio social.
\end{abstract}

PALAVRAS-CHAVE: Sexismo. Docência Superior. Disparidade. Representatividade Feminina.

\section{SEXISM AND TEACHING OF HIGHER EDUCATION: ANALYSIS OF FEMININE REPRESENTATIVENESS IN THE TEACHING OF EXACTING COURSES}

\begin{abstract}
This study aims to analyze the female representation in the faculty of the courses of exact, reflecting on the gender relations constituted in society that contribute to an inequality between men and women. In order to undertake such a discussion, we use as a theoretical contribution Barreto (2014), Butler (2012), Pinto e Cruz (2012), Lopes (2016), among others. We opted for a qualitative research of interpretative bibliographical search in search of meanings for the theoretical conceptions exposed in the existing literature. The analysis pointed out that despite the inclusion of several public policies aimed at higher education access, especially to the exact courses, over the last decades, that the participation of the female public in courses in this area is still very small, but that there is a growing therefore, research in this sense becomes important in the academic field to promote debate and reflection on gender inequality in our social environment.
\end{abstract}

KEYWORDS: Sexism. Higher Teaching. Disparity. Female Representation.

SEXISMO Y DOCENCIA DE LA ENSEÑANZA SUPERIOR: ANÁLISIS DE LA REPRESENTATIVIDAD FEMENINA EN LA DOCENCIA DE LOS CURSOS DE EXACTA 


\section{RESUMEN}

Este trabajo tiene por objetivo analizar la representatividad femenina en el cuerpo docente de los cursos de exactos, reflexionando acerca de las relaciones de género constituidas en la sociedad que contribuyen a una desigualdad entre hombres y mujeres. Para emprender tal discusión, utilizamos como aporte teórico Barreto (2014), Butler (2012), Pinto y Cruz (2012), Lopes (2016), entre otros. Optamos por una investigación cualitativa de cuño bibliográfico interpretativa en la búsqueda de significaciones para las concepciones teóricas expuestas en la literatura existente. Los análisis apuntaron que a pesar de la inserción a lo largo de las últimas décadas de diversas políticas públicas dirigidas al acceso de la enseñanza superior, en especial a los cursos de exactas, que la participación del público femenino en cursos de esa área todavía es bastante reducida, pero que hay una creciente $\mathrm{y}$, por eso, investigaciones en ese sentido se vuelven importantes en el ámbito académico para promover el debate y la reflexión acerca de la desigualdad de géneros existente en nuestro medio social.

PALABRAS CLAVES:Sexismo. La Docencia Superior. Disparidad. Representatividad Femenina 


\section{INTRODUÇÃO}

Vivemos em uma sociedade marcada pela desigualdade de gêneros, na qual muitos preconceitos permanecem nas entrelinhas. Os altos índices de violência contra mulher e a disparidade entre os salários de mulheres e homens, são alguns deles. Apesar da inserção das mulheres em cargos de poderes, advinda de fortes lutas travadas principalmente pelos movimentos feministas a desigualdade no tratamento sobre a questão de gêneros ainda é algo muito perceptível.

O movimento das mulheres no cenário social brasileiro nos últimos anos tem sido inquestionável na luta contra a opressão (SOARES, 1998). As mulheres sofrem com seu acesso limitado aos papéis e funções sociais. É inegável que a luta e a participação feminina nos movimentos sociais de uma sociedade marcada pela desigualdade e pela exclusão exercem um papel importante no questionamento do problema da disparidade de gênero (SOUZA-LOBO, 1991).

Nessa perspectiva, a educação configura-se como um dos principais eixos para a construção de uma sociedade igualitária entre mulheres e homens e para emancipação feminina (BRASIL, 2013). Sendo assim, torna-se de grande relevância social discutir sobre a perspectiva feminista e a luta das mulheres no contexto educacional. Visto que a educação desempenha um importante papel nas representações sociais e na construção de valores, então torna-se de extrema relevância dialogar sobre a questão da desigualdade de gênero no ambiente acadêmico. Investigar essa temática nesse espaço contribui para minimizar as desigualdades, tendo em vista que esse é um espaço de ensino-aprendizagem.

Com isso, buscou-se neste trabalho verificar a representatividade das mulheres na docência do ensino superior com enfoque para os cursos de ciências exatas, tendo em vista que apesar do aumento progressivo de mulheres nesses cursos, ainda se caracteriza por um espaço predominante masculino. Produziu-se um material para estudo e informativo, pois discussões como essas tornam-se fundamentais para questionar o problema da disparidade de gênero e contribuir para uma transformação social.

O trabalho está dividido nos seguintes itens: no primeiro, traçou-se reflexões acerca das desigualdades e diferenças de gêneros e sobre o sexismo, o segundo, apresenta uma breve discussão sobre a relação de gênero com a escolha do curso superior, em um terceiro momento discutiu-se sobre a representatividade feminina nas 
ciências exatas através de uma breve revisão literária e por fim apontou-se considerações finais com reflexões acerca das aprendizagens construídos ao longo desse processo da pesquisa.

\section{REFLEXÕES SOBRE GÊNERO E SEXISMO}

Os estudos sobre gênero e sexualidade têm uma importante finalidade educacional e demonstram que o conhecimento das interações entre homens e mulheres é algo fundamental para compreensão das relações sociais existentes. O conceito de gênero refere-se a um conjunto de práticas sociais que estabelecem grandes diferenças entre o que é entendido como feminino ou como masculino, e ainda indica comparações com relação às características e comportamentos de um em relação ao outro. (BARRETO, 2014).

Nessa perspectiva, entende-se que as reflexões sobre gênero e sexualidade são bem mais amplas do que as tratadas neste trabalho, porém o que se pretende são tecer breves considerações acerca das diferenças que são estabelecidas entre o que seria conferido ao gênero feminino e o que seria atribuído ao masculino na constante busca por dizimar aos poucos esses paradigmas e estereótipos previamente estabelecidos para cada gênero.

A Constituição Brasileira (1988) afirma no artigo $5^{\circ}$ do parágrafo $1^{\circ}$ que homens e mulheres são iguais em direitos e obrigações, todavia em muitos momentos da nossa história as mulheres tiveram que travar verdadeiras lutas em busca dos seus direitos. Um dos principais agravantes para a ocorrência desse fator se configura na questão da desigualdade de gênero, e na omissão pelos diversos tipos de seguimentos da sociedade desse tipo de descriminação sofrida pelas mulheres, o que pode acarretar em consequências gravíssimas como a violência, a exclusão e até mesmo a segregação das mulheres que acabam por se sentirem oprimidas e vulneráveis a esse verdadeiro problema social.

O Plano Nacional de Políticas para as Mulheres traz que na luta das mulheres ao longo da história, boa parte deu atenção para o processo educacional, com o intuito de demonstrar como as desigualdades entre mulheres e homens são reproduzidas no âmbito educacional. Deste modo, compreendendo a educação como importante mecanismo de transformação da realidade social, e reivindicando acesso igualitário para todas as 
pessoas. O Plano Nacional de Políticas para as Mulheres, no Brasil, trata a educação como um dos eixos fundamentais para a construção de uma sociedade igualitária entre mulheres e homens e para emancipação feminina (BRASIL, 2013).

Compreender o motivo para a desigualdade não é uma tarefa fácil, pois essa problemática está expressa nas mais diversas situações, dessa forma, o sexismo se caracteriza por essa ideia de inferioridade e superioridade ligada à questão do sexo que se expressa em toda atitude de descriminação em relação aos homens e as mulheres. $\mathrm{O}$ sexismo se trata de uma separação exclusiva entre o gênero masculino e feminino, no estabelecimento de relações de poder desiguais e à associação de significados pejorativos a um dos gêneros. (MADUREIRA, 2007).

Cabral (1998, p.1) descreve as relações de gênero como sendo "produto de um processo pedagógico que se inicia no nascimento e continua ao longo de toda a vida, reforçando a desigualdade existente entre homens e mulheres, principalmente em torno a quatro eixos: a sexualidade, a reprodução, a divisão sexual do trabalho e o âmbito público/cidadania”. Deste modo, pode-se concluir que o âmbito acadêmico é um lugar importante para ser trabalhado a questão de gênero, tendo em vista que o mesmo desempenha um papel fundamental para a construção de uma sociedade igualitária entre mulheres e homens.

\section{A RELAÇÃO DE GÊNERO COM A ESCOLHA DO CURSO SUPERIOR}

Torna-se proeminente realizar discussões que envolvam a relação de gênero com a escolha do curso de ensino superior, para que se possam entender por que há essa disparidade no espaço acadêmico, e compreender o porquê as mulheres estão mais presentes nos cursos de humanas e em contrapartida há um maior número de homens nos cursos de exatas. Pois, ao comparar o número de mulheres que ingressam em cursos dessas áreas esse aspecto é bem perceptível, desde a escolha pela graduação onde elas optam mais pela escolha de cursos das Ciências Humanas, Ciências Sociais, Ciências da Educação e Ciências da Saúde, enquanto eles buscam mais os cursos das Ciências Exatas e Tecnológicas de acordo com o INEP (2013, apud Pinto, Carvalho e Rabay, 2014). Desse modo, ainda conforme as autoras esse fator se justifica devido as questões sociais e culturais que cercam homens e mulheres desde o nascimento ao convívio 
social o que influenciaria de forma direta nas escolhas realizadas na vida adulta, inclusive na carreira profissional que pretendem seguir como destacado,

Refletir sobre a segmentação e desigualdade por sexo no processo de escolha de curso superior implica compreender o contexto cultural em que estamos inseridos/as, as influências da socialização na construção de projetos profissionais e a maneira como as pessoas naturalizam as diferenças sociais, já que mulheres e homens são tratados de forma diferente desde o nascimento em função do sexo biológico. (PINTO, CARVALHO e RABAY, 2014, p. 234).

Nesse sentido, é por meio dessa socialização realizada nos mais diversos espaços de convívio social que vão se diferenciando os modos de pensar, de ser e de agir, ou seja, o processo de formação emocional e psicológica de homens e mulheres que acabam por adquirir, valores, costumes e características diferentes, as mulheres em sua maioria parece estar mais envolvidas com causas humanitárias por estarem mais preocupadas com o próximo, com os problemas de cunho social e que envolvem o humano, enquanto que os homens em sua maioria possuem um pensamento mais técnico e prático, se detendo ao que pode ser comprovado e encontrado por meio de cálculos e estatísticas e isso acaba por diferenciar as escolhas de cada um, e em que víeis irão se deter em suas vidas profissionais.

Ainda nesse sentido Barretos (2014) destaca com relação as questões sociais, estas continuam marcadas pelas desigualdades, quando se trata de gêneros, inclusive com relação a divisão de tarefas,

“[...] em que, para determinadas posições, carreiras e funções, são valorizadas características atribuídas aos homens e à masculinidade e, para outras, características atribuídas às mulheres e ao feminino". Para uma boa atuação no mercado de trabalho, por exemplo, é ainda comum que se valorizem a racionalidade, a competitividade, a busca pelo sucesso, entendidas pelo senso comum como pertinentes ao universo masculino. Já os cuidados da casa e da família, como a valorização do amor, [...], da empatia, ainda são vistos muitas vezes como características [...] femininas. (BARRETOS 2014, p. 9 - 10).

Dessa forma, diante do supracitado entende-se que muitas vezes essa relação pela escolha do curso em que as mulheres irão ingressar e posteriormente atuar, muito se dá devido as questões sociais que envolvem os estereótipos designados ao que se remete ao universo feminino e ao que se remete ao universo masculino, e isso se reflete 
fortemente no mercado de trabalho e nos mais diversos âmbitos sociais, incluindo a área da educação, do ensino superior e na escolha pelos cursos.

\section{A REPRESENTATIVIDADE FEMININA NAS CIÊNCIAS EXATAS}

Historicamente a ciência sempre foi associada a uma atividade destinada ao sexo masculino, por muito tempo essa concepção se perpetuou como comprovam os dados de diversas pesquisas nesse sentido, as vagas nos cursos de ciências exatas eram predominantemente ocupadas por sujeitos do sexo masculino, entretanto, nas últimas décadas essa situação vem aos poucos sendo modificada.

Segundo Cooper et al (2010, apud PINTO e AMORIM, 2015, p.2), para que essa crescente continue e que se efetive de fato uma maior participação feminina em cursos onde predominam a participação masculina é necessária uma mudança de atitudes e comportamentos no âmbito acadêmico para se promover a equidade, para tanto é preciso que sejam pensadas políticas voltadas ao combate a descriminação e ao preconceito.

Em um estudo realizado por Pinto e Amorim (2015) sobre gênero e educação superior as autoras destacam que esse déficit do público feminino em cursos da área de exatas ainda é bem maior em cursos de Física, que é um dos campos da educação que contam com o crescimento ainda menor em comparação a outros cursos de exatas, como no caso dos cursos de Engenharias que antes também eram áreas dominadas pelo público masculino e hoje contam com o ingresso mais significativo do número de mulheres.

Na maioria dos países, menos de $20 \%$ dos títulos de doutorado em Física são de mulheres. No nível de graduação, a Turquia é o país com maior porcentagem de mulheres, correspondente a 39\%. Na Europa e nos Estados Unidos, embora a participação feminina nas Ciências Exatas esteja aumentando, a Física permanece como um campo masculinizado. No Brasil, assim como na maioria dos países em desenvolvimento que nos últimos 100 anos contaram apenas com $10 \%$ a 12\% de mulheres na Física [...] (PINTO e AMORIM, 2015, p. 3).

De acordo com Lopes (2016) essa participação feminina na docência tende a crescer, pois a presença de mulheres lecionando em cursos de exatas possibilita o estímulo através do exemplo para as novas gerações “O ser humano aprende, dentre 
outras formas, por meio da observação, e uma vez que as alunas interagem em um curso diretamente com professoras, elas passam a se reconhecer mais naquela futura profissão [...]” (LOPES, 2016, p. 3), pois as alunas acadêmicas passam a se reconhecer nesse papel docente e desfazer-se da ideologia hegemônica de que Física é um curso voltado para o público masculino.

É notório que a representatividade feminina nos mais diversos espaços de atuação social vem crescendo, e nos Cursos de Exatas não é diferente apesar de isso ocorrer de uma forma bem tímida. Lopes (2016) afirma que as investigações sobre tal temática buscam contribuírem para a diminuição da sub-representação feminina visando às políticas públicas de acesso ao ensino superior por parte do público feminino. Todavia, para que estas sejam implementadas é preciso que elas sejam antecipadas por meio de investigações que quantifiquem essa participação para que assim se possam materializar através dos dados as desigualdades existentes.

Ao longo da história muitas foram as lutas que as mulheres tiveram que enfrentar para terem mais espaço nas mais diversas áreas da sociedade, sobretudo nos campos que sempre tiveram por predomínio a representação masculina, e foi também transcendendo as mais diversas barreiras do preconceito, da descriminação e muitas vezes de atos violentos que a mulher vem aos poucos conquistando seu lugar em cargos de liderança e melhores posições sociais, ocupando funções importantes nos mais diversos setores como na política, na medicina, na astronomia, nas descobertas cientificas e tecnológicas, e isso também se reflete na educação, onde a mulher tem se destacado por estar em maior número em comparação aos homens nos bancos das universidades como destacado por Barretos (2014), o que posteriormente resultará em um maior número de engenheiras, advogadas, medicas e também professores formadas.

Contudo, há bem pouco tempo atrás esse quadro era diferente, pois quando se tratava do ensino superior o número de homens tanto alunos quanto professores dos cursos de ensino superior se sobressaiam em relação às mulheres tendo em vista que o ensino superior era um privilégio quase que exclusivamente dos homens, como afirma Barretos (2014). Todavia, esse quadro foi se modificando ao longo do tempo e hoje há um número mais compatível de homens e mulheres que estudam e lecionam nos cursos de ensino superior.

Nessa perspectiva, ainda de acordo com a autora, não se pode negar a maior representatividade que a presença feminina tem significativamente alcançado nos 
últimos anos nos cursos de nível superior no Brasil, todavia essa situação ainda é bem agravante quando se trata dos cursos das áreas de exatas, onde ainda há um número superior de homens em comparação as mulheres como assinalado na seguinte passagem: "É notório que historicamente a mulher conquistou espaços que antes só faziam parte os homens, mas sua inserção e permanência nas áreas de conhecimento pertencentes às exatas ainda não foram atingidas em condições de igualdade e reconhecimento. [...].” (SANTOS e RADL-PHILPP, 2015, p. 9).

Trazendo o estudo para o âmbito da Paraíba, Lopes, Lopes e Santos (2016), fizeram uma investigação sobre a representatividade das mulheres na docência do curso de física de duas instituições tradicionais do estado, o Curso de Física da Universidade Federal da Paraíba (UFPB) - Campus João Pessoa (JP) e o Curso de Física da UFCG Campus Campina Grande (CG) e Campus Cajazeiras (CZ). Os resultados apresentados no estudo das autoras demonstraram que aproximadamente $16,7 \%$ do quadro de professores são compostos por mulheres no Curso de Licenciatura em Física da UFCG - CZ, e nos cursos de Física da UFCG - CG e UFPB - JP, respectivamente a 12,1\% e $5,8 \%$. Em relação à média mundial que é de aproximadamente 10 a $12 \%$, elas concluíram que esta realidade, que ainda não é satisfatória, apresenta um quadro melhor do que as estatísticas globais.

É percebido que a equidade de gênero na docência dos cursos de exatas ainda não é uma realidade. Os percentuais demonstram que é necessário mais incentivo para que mulheres ocupem esses espaços, e quebrem as barreiras do machismo e sexismo presentes na sociedade. 


\section{CONSIDERAÇÕES FINAIS}

Os movimentos de luta das mulheres em busca de cessar essas diferenças de gênero na sociedade é algo antigo que transcendem gerações onde estas buscam além de direitos iguais, mais reconhecimento e valorização, obtendo com isso uma maior representatividade nos mais diversos âmbitos sociais. Esse ativismo feminista proporcionou a conquista de muitos direitos para as mulheres, além de contribuir para o crescimento da conscientização delas para reivindicarem por igualdades de direitos. Mas, mesmo com todo esse avanço muitos preconceitos ainda existem, como em relação a distinção das carreiras profissionais seguidas por homens e mulheres e nas disparidades salariais existentes delas em comparação a eles.

Atualmente no Brasil há uma política pública direcionada aos direitos das mulheres, buscando fortalecer esse processo de igualdades de gênero entre homens e mulheres e seria fundamental que aliado a essas políticas e aos movimentos, houvesse uma maior discussão nas mídias, nas escolas e nas instituições de ensino superior de modo que cada vez mais alcance um número maior de pessoas para que dessa forma esses movimentos ganhassem mais força promovendo o protagonismo das mulheres na sociedade e a garantia da conquista de seus direitos.

O presente estudo foi um resultado de pesquisas realizadas nas literaturas já existentes sobre a temática, onde procurou-se realizar uma discussão de forma reflexiva, destaca-se que não se trata de um estudo conclusivo sobre sexismo e docência até porque acredita-se que novas pesquisas precisam e devem ser realizadas como forma de possibilitar uma maior reflexão e consequente entendimento acerca do tema. No entanto, fica esta como incitação à continuidade do debate. 


\section{REFERÊNCIAS BIBLIOGRÁFICAS}

Plano Nacional de Políticas para as Mulheres. Brasília, DF, 2013.

. Secretária de Políticas para as mulheres. Brasília, DF, 2015.

BARRETO, A. A Mulher no Ensino Superior: Distribuição e Representatividade. Cadernos do GEA, n. 6, 2014.

BRASIL. Constituição da República Federativa do Brasil. Brasília, DF, 1988.

BUTLER, J. Problemas de gênero: feminismo e subversão da identidade. Tradução de Renato Aguiar. 4. ed. Rio de Janeiro: Civilização Brasileira, 2012.

CABRAL, F.; DÍAZ, M. Relações de gênero. Secretaria Municipal de Educação de Belo Horizonte; Fundação Odebrecht. Cadernos afetividade e sexualidade na educação: um novo olhar. Belo Horizonte: Gráfica e Editora RonaLtda, 1998. p. 142150 .

LOPES, M. D.; LOPES, G. R.; DOS SANTOS, J. A. Um Ensaio Sobre A Representatividade Feminina No Corpo Docente Do Curso De Física Da Cidade De Cajazeiras/Pb. Disponível em: https://editorarealize.com.br/revistas/conidis/trabalhos/TRABALHO_EV064_MD4_SA 13_ID1036_24102016012410.pdf> Acesso em: 14 jan. 2019.

LOPES, M. D.;LOPES. G. R.; SANTOS. J. A. dos.Um Ensaio sobre a Representatividade Feminina no Corpo Docente do Curso de Física da Cidade de Cajazeiras/PB. In: I CONGRESSO INTERNACIONAL DA DIVERSIDADE DO SEMIÁRIDO.2016, Campina Grande. Anais... I CONIDIS, 2016. Disponível em:<http://www.editorarealize.com.br/revistas/conidis/trabalhos/TRABALHO_EV064_ MD4_SA13_ID1036_24102016012410.pdf> Acesso em: 9 jan. 2019.

MADUREIRA, A. F. A. Gênero, sexualidade e diversidade na escola: A construção de uma cultura democrática. Tese de Doutorado. Universidade de Brasília, 2007. Disponível em: <http://repositorio.unb.br/handle/10482/1610> Acesso em: 14 jan. 2019.

PINTO, É. J. S.; AMORIM, V. G.Gênero e Educação Superior: Um Estudo sobre as Mulheres na Física.37 Reunião Nacional da ANPEd -UFSC - Florianópolis, out. 2015. Disponível em: <http://37reuniao.anped.org.br/wp-content/uploads/2015/02/TrabalhoGT23-3778.pdf> Acesso em: 18 jan. 2019.

PINTO, É. J. S.; CARVALHO, M. E. P. de.; RABAY, G. Gênero:um fator condicionante nas escolhas de cursos superiores. In:18 REDOR,Universidade Federal Rural de Pernambuco,Recife - PE. Anais Eletrônicos.2014, p. 233-249. Disponível em:<https://paradoxzero.com/zero/redor/wp-content/uploads/2015/04/1893-4523-1PB.pdf> Acesso em: 15 jan. 2019. 
PINTO, M. D. F.; CRUZ, M. H. S. Mapeando diferenças de gênero no ensino superior da Universidade Federal de Sergipe. São Cristóvão: Editora UFS, 2012.

SANTOS, C. S.; RADL-PHILPP, R. M. Mulheres nas Ciências Exatas: Políticas Públicas de Acesso ao Ensino Superior. 2015. Disponível em:<http://www.uneb.br/enlacandosexualidades/files/2015/07/COMUNICA\%C3\%87\% C3\%83O-SANTOS.RADL1.pdf> Acesso em: 19 jan. 2019.

SOARES, V. Muitas faces do feminismo no Brasil. Mulher e Política: Gênero e feminismo no Partido dos Trabalhadores. SP, Fundação Perseu Abramo, 1998.

SOUZA-LOBO, E. O gênero da representação: Movimento de mulheres e representação política no Brasil (1980-1990). Revista Brasileira de Ciências Sociais, v. 17, p. 7-14, 1991. 